\title{
HEALTH AND SAFETY INSPECTORS' PERCEPTIONS OF PREVENTABILITY OF WORK-RELATED ILL HEALTH AND INJURY
}

Gillian Frost,' Simon Clarke2 ${ }^{1}$ Health \& Safety Laboratory, Buxton, UK; ${ }^{2}$ Health \& Safety Executive, Bootle, UK

10.1136/oemed-2011-100382.6

Objectives A project is underway to assess the burden of workrelated ill health and injury (harm) due to current occupational exposures in GB, and its outputs will help to inform prioritisation of regulatory health and safety activity. However, not all work-related harm is equally preventable. The study reported here gathered information on regulatory inspectors' perceptions of the preventability of a range of work-related harm in terms of two factors: 1) possible impact of additional regulatory activity, and 2) dependency of the level of harm on current regulatory activity.

Methods Fourteen discussion groups involving 88 health and safety inspectors were held between July 2008 and March 2009. Participants completed a questionnaire designed to gauge their opinion on the two preventability factors for a range of industries and work-related harm. Results were fed back to participants, who could then discuss and change their responses.

Results The inspectors identified stress as having the lowest dependency on current regulatory health and safety activity of all work-related harm, and the lowest possibility of further impact. Falls from height, and workplace transport incidents had the greatest dependency on current activity, and the greatest possibility of further impact. The agricultural industry had the lowest possibility of further impact, with Manufacturing and Construction having the greatest dependency on current activity.

Conclusions This study developed simple methodology to gather information on inspectors' perceptions of preventability. The exercise could be repeated in different study 
populations in order to gauge the robustness of results. (C) Crown Copyright (2011). 